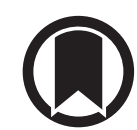

CrossMark

\section{Added value of chest computed tomography in suspected COVID-19: an analysis of 239 patients}

\author{
To the Editor:
}

Since the outbreak of the coronavirus disease 2019 (COVID-19) pandemic, clinicians have been struggling with the optimal diagnostic approach of suspected patients. Real-time reverse transcriptase-polymerase chain reaction (RT-PCR) testing of respiratory samples is generally being considered as the reference standard for establishing severe acute respiratory syndrome coronavirus 2 (SARS-CoV-2) infection [1]. However, RT-PCR results take hours to become available and, although highly specific, sensitivity is moderate [2-4]. This could result in delayed and suboptimal clinical decision making.

Several reports have suggested a potential role for chest computed tomography (CT) in patients with suspected COVID-19 [5-10]. Major advantages of CT are that it provides immediate results and can identify alternative diagnoses for respiratory symptoms. CT may show signs suspicious or typical of COVID-19 in patients with a negative RT-PCR result, ensuring that the patient remains isolated. On the other hand, a negative CT result in combination with a negative RT-PCR result may exclude COVID-19 with a higher level of certainty, in which case patient de-isolation can be considered, and additional diagnostic work-up can be initiated. In this study, we evaluated the added value of chest CT over RT-PCR testing alone.

Consecutive patients with suspected COVID-19 who presented to the emergency department of our university hospital (Academic Medical Center, Amsterdam University Medical Centers, the Netherlands) from 16 March to 16 April 2020 were retrospectively assessed for inclusion. Patients with suspected COVID-19 were those with 1) fever, 2) cough or dyspnoea, or 3) other signs suggestive of COVID-19 (e.g. gastro-intestinal symptoms). Patients were included if they were 18 years or older, required hospital admission, and underwent both chest CT and RT-PCR testing for SARS-CoV-2 infection upon admission. In our hospital, performing both these tests is standard practice for patients with suspected COVID-19 requiring admission. We excluded patients who already had a prior positive RT-PCR result. Data were extracted from patient records by one author (D.A. Korevaar, R.S. Kootte or L.P. Smits).

A non-enhanced low-dose chest CT scan was obtained from all patients (Somatom Force, Siemens Healthineers, Forchheim, Germany). CT images were read as part of standard clinical practice by attending radiologists, with varying degrees of experience. To improve uniformity, an informal second read was performed in some cases by a dedicated acute radiologist, and disagreements were solved by consensus. The radiological probability of pulmonary manifestations of COVID-19 was reported based on the "CO-RADS classification", a standardised reporting system for patients with suspected COVID-19, ranging from 1 (very unlikely) to 5 (very likely) [11, 12]. In this study, CO-RADS scores of 1-2 were considered as negative, scores of $4-5$ were positive, and a score of 3 was indeterminate. CT readers were not blinded to clinical information, but RT-PCR results were never available at the time of reading. If CT reports did not contain a CO-RADS score $(n=20)$, images were re-read by a radiologist blinded to the RT-PCR results (L.F.M. Beenen).

Nose and throat swabs, and, if possible, sputum samples, were obtained for RT-PCR testing of SARS-CoV-2 infection [13]. When the initial RT-PCR result was negative or indeterminate, but clinical suspicion of COVID-19 remained, repeat RT-PCR testing was generally performed, most often within the first $24 \mathrm{~h}$ after admission. In this study, patients with a positive initial or repeat RT-PCR result within

@ERSpublications

In a high-prevalence emergency department setting, chest CT showed a high probability of COVID-19 in $30 \%$ of patients with a negative or indeterminate initial RT-PCR result https://bit.ly/38hLDzR

Cite this article as: Korevaar DA, Kootte RS, Smits LP, et al. Added value of chest computed tomography in suspected COVID-19: an analysis of 239 patients. Eur Respir J 2020; 56: 2001377 [https://doi.org/ 10.1183/13993003.01377-2020]. 
7 days after admission were considered to have COVID-19, whereas patients who only had negative RT-PCR results and a CO-RADS score of 1-3 were considered not to have COVID-19. For patients who had negative RT-PCR results but a CO-RADS score of 4-5, serological testing for SARS-CoV-2 antibodies was performed if serum was available (Biozek, COVID-19 IgG/IgM rapid test), and the final diagnosis was considered as "COVID-19 very likely" if this was positive. If no serum was available or serology was negative, all the available clinical information (including additional microbiological tests) was assessed in a multidisciplinary meeting including two pulmonologists (P.I. Bonta, J.T. Annema), an infectiologist (A. Goorhuis), a virologist (J. Schinkel) and a radiologist (L.F.M. Beenen), to reach consensus about the final diagnosis, classified as "COVID-19 very likely", "uncertain, or "COVID-19 very unlikely". This meeting was held 7 days after admission of the last patient in the study.

The primary outcome was the proportion of patients with a negative initial RT-PCR result who had a positive chest CT result (i.e. CO-RADS 4-5). The full study protocol is available upon request.

During the study period, 258 patients with suspected COVID-19 were admitted, of which 19 were excluded because they had a prior positive RT-PCR result $(n=8)$, no RT-PCR testing was performed $(n=2)$, or no chest CT was performed $(n=9)$. The remaining 239 patients underwent both CT and RT-PCR testing upon admission and were included. Median age of patients was 63 years (interquartile range 51-71 years), $139(58.2 \%)$ were male, median time since symptom onset was 7 days (interquartile range 3-10 days), and 18 (7.5\%) were immediately admitted to ICU, whereas $22(9.2 \%)$ were transferred to ICU after initial ward admission. Overall, 112 patients (46.9\%) had a positive initial RT-PCR result, and 14 (5.9\%) had a positive repeat RT-PCR result within 7 days after admission. Additional microbiological tests included blood culture in 206 patients ( $n=8$ positive), sputum culture in 85 ( $n=12$ positive), PCR testing for influenza virus and respiratory syncytial virus in respiratory samples in 149 ( $n=2$ and $n=1$ positive, respectively), PCR testing for other common respiratory viruses in 25 ( $\mathrm{n}=1$ positive), pneumococcal urinary antigen testing in 92 ( $\mathrm{n}=1$ positive), Legionella urinary antigen testing in 127 ( $\mathrm{n}=1$ positive), and PCR testing for atypical respiratory pathogens in 34 ( $n=0$ positive).

Chest CT results stratified by RT-PCR results are reported in the table 1 . Of 127 patients with a negative or indeterminate initial RT-PCR result, 38 (29.9\%) had a positive CT result (CO-RADS 4-5). Of these, 13 had a positive RT-PCR result upon repeat testing. Serological testing could be performed in six of the other 25 patients, and was positive for SARS-CoV-2 antibodies in five of them. The remaining 20 patients were assessed in a multidisciplinary consensus meeting. For 13 of them it was concluded that COVID-19 was "very likely", for three that COVID-19 diagnosis was "uncertain" (one with negative serological testing), and for four that COVID-19 was "very unlikely"; in three out of these four patients an alternative diagnosis was found: heart failure, bacterial pneumonia and rhinovirus pneumonia. Overall, of all 143 patients with a final diagnosis of COVID-19 after at least 7 days of follow-up, 18 (12.6\%) had a negative initial or repeat RT-PCR result, but had a positive CT result (CO-RADS 4-5).

Of patients with a negative or indeterminate initial RT-PCR result, 65 (51.2\%) had a negative CT result (CO-RADS 1-2), and $24(18.9 \%)$ had an indeterminate CT result (CO-RADS 3). In 42 of these 89 patients, RT-PCR testing was repeated at least one time within the first 7 days after admission, and only one became positive. Of 112 patients with a positive initial RT-PCR result, CT was positive (CO-RADS 4-5) in 104 (92.9\%), indeterminate (CO-RADS 3) in five (4.5\%), and negative (CO-RADS 1-2) in three (2.7\%).

\begin{tabular}{|c|c|c|c|}
\hline & $\begin{array}{l}\text { Initial RT-PCR: } \\
\text { positive }\end{array}$ & $\begin{array}{c}\text { Initial RT-PCR: } \\
\text { negative/non-interpretable }\end{array}$ & \\
\hline $\begin{array}{l}\text { Chest CT: CO-RADS 1-2 } \\
\text { (COVID-19 unlikely) }\end{array}$ & $\begin{array}{c}3 \\
2.7 \%(95 \% \mathrm{Cl} 0.0-7.3 \%)\end{array}$ & $\begin{array}{c}65^{\#} \\
51.2 \%(95 \% \mathrm{Cl} 42.5-60.6 \%)\end{array}$ & 68 \\
\hline $\begin{array}{l}\text { Chest CT: CO-RADS } 3 \\
\text { (COVID-19 indeterminate) }\end{array}$ & $\begin{array}{c}5 \\
4.5 \%(95 \% \mathrm{Cl} 0.9-9.1 \%)\end{array}$ & $\begin{array}{c}24^{\text {9 }} \\
18.9 \% \text { (95\% Cl } 10.2-28.3 \%)\end{array}$ & 29 \\
\hline $\begin{array}{l}\text { Chest CT: CO-RADS 4-5 } \\
\text { (COVID-19 likely) }\end{array}$ & $\begin{array}{c}104 \\
92.9 \%(95 \% \mathrm{Cl} 89.3-97.5 \%) \\
112 \\
(100 \%)\end{array}$ & $\begin{array}{c}38^{+} \\
29.9 \%(95 \% \mathrm{Cl} 21.3-39.3 \%) \\
127 \\
(100 \%)\end{array}$ & 239 \\
\hline
\end{tabular}

Data are presented as $\mathrm{n} \%(95 \% \mathrm{Cl})$, unless otherwise stated. \#: there were no "non-interpretable" initial RT-PCR results; 22 patients had at least one repeat RT-PCR, of which one was positive; ": one initial RT-PCR result was "non-interpretable"; 20 patients had at least one repeat RT-PCR, of which none were positive; ${ }^{+}$: seven initial RT-PCR results were "non-interpretable"; 35 patients had at least one repeat RT-PCR, of which 13 were positive. 
The retrospective nature of our study may be considered a limitation, but risk of selection bias is limited as only $4.4 \%$ of patients admitted with suspected COVID-19 were excluded because CT or RT-PCR testing was not performed. Although CT scans were scored by single readers with varying levels of experience, this reflects daily clinical practice. As there is currently no reliable reference standard for COVID-19, we had to rely on ruling-out other explanations and multidisciplinary consensus in patients with negative RT-PCR results, which may have led to false-positive CT results. Compared to our study, a much larger proportion of false-positive CT results against RT-PCR testing was identified by AI et al. [7] in their analysis of 1014 patients with suspected COVID-19, resulting in a specificity of only $25 \%$. This difference may be explained by the fact that we strictly applied a standardised reporting system (CO-RADS), and because their study was performed in the early phase of the pandemic; clinical knowledge on typical radiological findings in COVID-19 has increased tremendously since then and could be applied by radiologists in our hospital [14].

In conclusion, in a high-prevalence emergency department setting, we found that chest CT showed high probability of COVID-19 (CO-RADS 4-5) in $29.9 \%$ of patients with a negative or indeterminate initial RT-PCR result. As the majority of these patients $(81.6 \%)$ had a proven or "very likely" diagnosis of COVID-19 after repeat RT-PCR testing or clinical follow-up, we believe that CT helps in the identification of patients who should be admitted in isolation. CT showing low or intermediate probability of COVID-19 combined with a negative RT-PCR rarely resulted in a positive subsequent RT-PCR result and de-isolation in such patients may be considered. Based on these findings, we recommend the use of chest CT in combination with RT-PCR testing in patients with suspected COVID-19 in emergency departments, provided that adequate infection control protocols in CT suites are in place. Whether this strategy is superior to one that incorporates chest radiography in terms of accuracy, patient-important outcomes or costs is currently unknown and could be subject to future studies.

Daniël A. Korevaar ${ }^{1}$, Ruud S. Kootte ${ }^{2}$, Loek P. Smits ${ }^{2}$, Joost G. van den Aardweg ${ }^{1}$, Peter I. Bonta ${ }^{1}$, Janke Schinkel ${ }^{3}$, René M. Vigeveno ${ }^{3}$, Inge A.H. van den Berk ${ }^{4}$, Maeke J. Scheerder ${ }^{4}$, Bregtje A. Lemkes ${ }^{5}$, Abraham Goorhuis ${ }^{5}$, Ludo F.M. Beenen $\oplus^{4}$, Jouke T. Annema ${ }^{1}$ and on behalf of the Amsterdam UMC location AMC COVID-19 group ${ }^{6}$ ${ }^{1}$ Dept of Respiratory Medicine, Amsterdam University Medical Centres, University of Amsterdam, Amsterdam, the Netherlands. ${ }^{2}$ Dept of Internal Medicine, Amsterdam University Medical Centres, University of Amsterdam, Amsterdam, the Netherlands. ${ }^{3}$ Dept of Medical Microbiology and Infection Prevention, Amsterdam University Medical Centres, University of Amsterdam, Amsterdam, the Netherlands. ${ }^{4}$ Dept of Radiology, Amsterdam University Medical Centres, University of Amsterdam, Amsterdam, the Netherlands. ${ }^{5}$ Dept of Infectious Diseases, Amsterdam University Medical Centres, University of Amsterdam, Amsterdam, the Netherlands. ${ }^{6}$ All collaborators in the "Amsterdam UMC location AMC COVID-19 group" are listed in the acknowledgements section.

Correspondence: Daniël A. Korevaar, Dept of Respiratory Medicine, Amsterdam University Medical Centres, University of Amsterdam, Meibergdreef 9, 1105 AZ Amsterdam, the Netherlands. E-mail: d.a.korevaar@amsterdamumc.nl

Received: 24 April 2020 | Accepted after revision: 24 June 2020

Data availability: The full study protocol is available upon request.

Collaborators in the Amsterdam UMC location AMC COVID-19 group are: Josje Altenburg, Godelieve J. de Bree, Laurence M. Crombag, Marc R.W. Engelbrecht, Suzanne E. Geerlings, Tom van Gool, Martin P. Grobusch, Vanessa C. Harris, Thecla A.M. Hekker, Robert Hemke, Sabine M. Hermans, Jorrit J. Hofstra, Joppe W. Hovius, René E. Jonkers, Saskia Kolkman, Marnix P.M. Kop, Christof J. Majoor, Jeannine F. Nellen, Ikrame Oulad Abdennabi, Robrecht N. Planken, Tom van der Poll, Jan M. Prins, Adrienne van Randen, Peter Reiss, Charlotte A. van Ruitenbeek, Laura J. Schijf, Frank F. Smithuis, Veroniek Spoorenberg, Reindert P. van Steenwijk, Cornelis Stijnis, Femke Struik, Jeroen A.W. Tielbeek, Marc van der Valk, Anton Vonk Noordegraaf, Michèle van Vugt, Els J.M. Weersink, W. Joost Wiersinga, and Rens Zonneveld, all affiliated to the Amsterdam University Medical Centres, University of Amsterdam, the Netherlands.

Conflict of interest: None declared.

\section{References}

1 Cheng MP, Papenburg J, Desjardins M, et al. Diagnostic testing for severe acute respiratory syndrome-related coronavirus 2: a narrative review. Ann Intern Med 2020; 172: 726-734.

2 Long C, Xu H, Shen Q, et al. Diagnosis of the coronavirus disease (COVID-19): rRT-PCR or CT? Eur J Radiol 2020; $126: 108961$.

3 Xie X, Zhong Z, Zhao W, et al. Chest CT for typical 2019-nCoV pneumonia: relationship to negative RT-PCR testing. Radiology 2020; 296: E41-E45.

4 Wang W, Xu Y, Gao R, et al. Detection of SARS-CoV-2 in different types of clinical specimens. JAMA 2020; 323: 1843-1844.

5 Yang S, Shi Y, Lu H, et al. Clinical and CT features of early stage patients with COVID-19: a retrospective analysis of imported cases in Shanghai, China. Eur Respir J 2020; 55: 2000407.

6 Zhang S, Li H, Huang S, et al. High-resolution computed tomography features of 17 cases of coronavirus disease 2019 in Sichuan province, China. Eur Respir J 2020; 55: 2000334.

7 Ai T, Yang Z, Hou H, et al. Correlation of chest CT and RT-PCR testing in coronavirus disease 2019 (COVID-19) in China: a report of 1014 cases. Radiology 2020; 296: E32-E40. 
8 Rubin GD, Ryerson CJ, Haramati LB, et al. The role of chest imaging in patient management during the COVID-19 pandemic: a multinational consensus statement from the Fleischner Society. Chest 2020; 158: 106-116.

9 Pan Y, Guan H, Zhou S, et al. Initial CT findings and temporal changes in patients with the novel coronavirus pneumonia (2019-nCoV): a study of 63 patients in Wuhan, China. Eur Radiol 2020; 30: 3306-3309.

10 McInnes MD, Leeflang M, Salameh JP, et al. Imaging tests for the diagnosis of COVID-19 (protocol). Cochrane Database Syst Rev 2020: CD013639.

11 COVID working group of the Dutch Radiological Society. CO-RADS classification. https://radiologyassistant.nl/ chest/covid-19-corads-classification Date last updated: 25 March 2020.

12 Prokop M, van Everdingen W, van Rees Vellinga TL, et al. CO-RADS - a categorical CT assessment scheme for patients with suspected COVID-19: definition and evaluation. Radiology 2020; 296: E97-E104.

13 Corman VM, Landt O, Kaiser M, et al. Detection of 2019 novel coronavirus (2019-nCoV) by real-time RT-PCR Euro Surveill 2020; 25: 2000045.

14 Ojha V, Mani A, Pandey NN, et al. CT in coronavirus disease 2019 (COVID-19): a systematic review of chest CT findings in 4410 adult patients. Eur Radiol 2020; in press [https://doi.org/10.1007/s00330-020-06975-7].

Copyright (C)ERS 2020 .

This version is distributed under the terms of the Creative Commons Attribution Non-Commercial Licence 4.0. 